\title{
Primitive Theories of Religion: Evolutionism after Evans-Pritchard
}

\author{
Konrad Talmont-Kaminski \\ Society \& Cognition Unit, University of Bialystok
}

\begin{abstract}
In 1965 Evans-Pritchard published what is considered the ultimate critique of evolutionist theories of religion. More than five decades later, evolutionary approaches to the study of religion are thriving. Is this because the lessons contained in Theories of Primitive Religion have been forgotten? Or is it that the modern approaches have managed to learn from the errors Evans-Pritchard catalogued? The situation is somewhat more complex. It would be facile to dismiss the most fundamental criticisms of evolutionism made by Evans-Pritchard on the grounds that the 'evolutionary' theories he focused upon were based much more on Comte's positivist view of history than on Darwin's evolutionary theory and, therefore, have little in common with modern approaches that aim to be based upon current evolutionary biology. Evans-Pritchard's critique was much more thorough than that and concerned theory, methods and data - it applied far beyond evolutionist theories, to a broad selection of approaches. While enormous progress has been made concerning the available data, the variety of methods used to obtain it and the theories used to make sense of it, many of the issues EvansPritchard raised keep reappearing in novel forms. As such his book remains a significant reference point for any attempt to understand religion. It is not, however, the searing indictment of the use of evolutionary theory in the study of religion that some might wish for. The criticisms Evans-Pritchard raised affect theories of religion in general and it is the multidisciplinary approach presented by cognitive science of religion that is best placed to counter them, largely thanks to its use of evolutionary theory.
\end{abstract}

\section{Keywords}

evolutionism, cognitive science of religion, progressivism, reductionism

\section{Contact address}

Konrad Talmont-Kaminski, Society \& Cognition Unit, University of Bialystok, Plac NZS 1, 15-420

Bialystok, Poland; k.talmontkaminski@gmail.com 


\section{Acknowledgements}

This research was supported by grant no. 2017/27/B/HS1/02089 from the National Science Centre, Poland.

\section{Introduction}

In the first section of this paper, I look at Evans-Pritchard's (1965) basic critique of evolutionism as based upon a positivist view of human history as the progressive rejection of religion. He argues that this view is at best lacking in evidential support and, at worse, is actually contradicted by it. With the benefit of an extra five decades of evidence, the situation has not improved for such a view. Modern evolutionary approaches, however, do not accept the positivist view of history. In their case, the evolutionary view that lies at their heart is that of evolutionary biology, which is explicitly non-progressive in its assumptions. As such, the gist of Evans-Pritchard's critique does not affect these views. This is not to say that it is not relevant, however. First of all, the phenomenon of secularisation is not a figment of the theorist's imagination and, thus, still requires explanation. It is only that such an explanation as is given by the existential security thesis, for example, is provided in terms of the existence of particular social factors rather than in terms of an overarching historical narrative. Secondly, evolutionary approaches to understanding human cognition help us understand why it was that the positivist misunderstanding of Darwin's view of evolution was so attractive and in some quarters still remains so.

In the second section, I consider the broader thrust of Evans-Pritchard's critique - that theory in the study of religion has in general outstripped data. He makes this fundamental point time and again with regard to very many of the claims raised by his predecessors. Again, the situation has fundamentally altered in the years since he made his comments. In his book, Evans-Pritchard considers anthropological descriptions of a broad variety of societies as the empirical basis for theory-making regarding the nature of religion. Of course, the amount of this kind of evidence is now much greater than was available at his time. More fundamentally, however, this kind of data now represents only one kind of empirical evidence that researchers can work with to understand religion. Researchers within cognitive science of religion can rely upon quantitative sociological data, massive historical databases, experimental psychological laboratory studies as well as physiological data from the field, just to name some of the potential sources. The growth in the breadth of available data has also gone hand in hand with the development of theory and method. Yet, despite all of these vital developments, it would be false to claim that the general issue of theory outstripping data has disappeared. Indeed, Evans-Pritchard's use of the phrase "just-so stories" has much currency in evolutionary approaches to explaining religion. This is because of just how difficult it is to show that some particular behaviour is adaptive, as can be appreciated by considering the criteria first proposed by Niko Tinbergen. At the same time, however, science must have theories that run ahead of the evidence. The 
question is whether in particular cases this is justified and how the theories in question should be treated.

In the third section, I consider the fundamental similarities and differences between today's evolutionary approach and the theories criticised by Evans-Pritchard. The distinction between psychological and sociological accounts, that he uses in his book, is one that still is reflected within the modern approach. It plays a very different role, however, in today's study of religion because of explanatory pluralism having become the norm. This means that the issue of reductionism that Evans-Pritchard considers at length can be dealt with in a profoundly restructured context. Evans-Pritchard's own position is antireductionist. From the point of view of modern approaches, however, it appears to be based upon the assumption that what is sought by researchers is THE theory of religion. Modern approaches to understanding religion are cheerfully and pragmatically pluralist about the theories they employ as well as being at best agnostic as to whether the concept of 'religion' is robust enough to hang any substantive theorising upon it. In effect, modern approaches focus on theories of religious behaviours and beliefs that often rely upon more than one level of description, rather than an overarching account of religion itself. The overall approach contains the tools that make it possible to avoid Evans-Pritchard's anti-reductionist concerns.

\section{Evolutionism, old and new}

This is how Evans-Pritchard opens his discussion of psychological theories of religion:

"The theory of President de Brosses, a contemporary and correspondent of Voltaire, that religion originated in fetishism, was accepted until the middle of last century. The thesis, taken up by Comte, was that fetishism, the worship, according to Portuguese sailors, of inanimate things and of animals by the coastal Negroes of West Africa, developed into polytheism and polytheism into monotheism." (Evans-Pritchard 1965, 20)

This example contains the main two characteristics of evolutionary theories of religion as examined by Evans-Pritchard. The first of them is conjecture regarding the origin of religion. This conjecture is typically understood as a historical claim (although at times Evans-Pritchard appears to be talking about the very different issue of the psychological processes that make it likely that someone will come to believe existing religious claims). The second is a proposal regarding the stages through which religion has developed, this 'evolution' of religion being understood as progressive. Furthermore, both of the claims are very general in nature, suggesting that the claims have something of historical necessity to them - religion could only appear in the theorised way and must develop through the proposed stages in the proposed order.

Significantly, despite discussing evolutionist views at length, Evans-Pritchard does not discuss them in the light of Darwin's work on the theory of evolution. There is a very good reason for this - the approach he criticises has relatively little to do with Darwinian 
evolution or the biological theories that have been developed on that basis. Indeed, the one connection Evans-Pritchard makes is to present evolutionism as much more a descendant of the positivist view of history proposed by Comte. Just how distant this evolutionism is from the work of Darwin can be appreciated by considering how little the main elements listed above have to do with Darwin's view of biological evolution. It must, firstly, be noted that evolutionism concerns cultural changes, whereas Darwin concerned himself with biological change. The necessary broadening of the notion of evolution is in my view justifiable, but it is a change in the subject matter that needs to be noted. More importantly, Darwin is quite clear that his theory of evolution does not provide an explanation for how life appeared, but merely deals with how particular species have come to exist. It is The Origin of Species and not The Origin of Life, after all. By analogy, a 'Darwinian' theory of the evolution of religion would prima facie have nothing to say about the historical genesis of religion and, instead, would concern itself with how religions have changed over time, and especially with how new religions have come to form. This brings us to the third difference to be considered. Darwinian theory of evolution is explicitly undirected. Biological evolution is not progress. There is, in fact, no inherent direction to it. This runs counter to the evolutionist idea of there being a particular sequence of stages that religion goes through. Finally, Darwinian evolution is the result of countless incidents and random mutations which, were the clock wound back, would play out very differently. Unlike the evolutionism that Evans-Pritchard critiques, Darwinian evolution is definitely not a matter of fate revealing itself.

So, while Evans-Pritchard's characterisation of the early evolutionist views from the turn of the twentieth century is quite accurate, it does not fit modern evolutionary approaches to explaining religion. Modern approaches, including such as are developed within the broad scope of cognitive science of religion, reject as pseudoscientific the evolutionist views that Evans-Pritchard objected to. Instead, today's researchers look to the solid theoretical basis provided by modern evolutionary theory which has its historical roots in part in Darwin's work and holds much the same views on the questions of genesis and progress as he put forward. As such, Evans-Pritchard's critique of evolutionism has, at least prima facie, no hold on modern attempts to apply evolutionary theory to the understanding of religion. Indeed, given its basis in Darwin's work, cognitive science of religion could be said (tongue-in-cheek) to have agreed with Evans-Pritchard's critique a hundred years before he put it forward.

Evans-Pritchard's general critique is aimed much broader, however, than just at the principles of evolutionism. For this reason, it definitely needs to be considered by modern theorists, despite their rejection of anything like a positivist theory of history. EvansPritchard argues that evolutionist theories either run counter to available evidence, lack sufficient positive evidence or, in the case of claims about the genesis of religion, could not possibly be provided with any evidence. He makes much the same criticism of the many other theories of religion he considers. Time and again he points to the disconnect between theory and evidence within the study of religion. The question of whether such 
a disconnect exists in the case of modern evolutionary theories of religion has to, therefore, be asked and will be considered at length in the second section. Before we do, however, it is useful to consider what light has been thrown upon the original evolutionist views in the fifty or so years since Evans-Pritchard published his critique.

To the optimistically-inclined Europeans and Americans of the late nineteenth century from whose ranks the majority of evolutionist theorists came, the history of civilisation appeared to be a history of progress. It is hardly surprising, therefore, that their theories of religion assumed that progress, as understood by them, was the norm, especially given how little data they had readily available to them regarding other times and cultures. Our situation in both these respects is quite altered. The amount of historical and archaeological data we have is incomparably greater and shows that throughout history cultures have grown and collapsed, with many societies 'regressing' to a much more 'primitive' stage. Indeed, our own society now stands upon the brink of what could very well turn out to be another example of cultural collapse, brought on by our degradation of our environment and the overuse of the limited natural resources that are available to us. Yet, at the same time, within the confines of the cultures the evolutionist theorists came from, many changes have occurred that they would have been happy to point to as evidence for progress. The primary one to consider, that has fundamentally altered the societies of developed countries in the last hundred years, is secularisation.

At the time that Comte proposed his view that science would replace religion as the basis for society there were no countries in which nonbelievers were anything other than a relatively small minority. Now, quantitative sociologists are observing a steady drop in religious affiliation throughout the developed countries (Voas 2008) with the numbers of self-declared atheists and agnostics having reached half or more of the population in countries such as France, Germany, the Czech Republic, Denmark and Japan. Even the US, which has for a long time failed to show the same trend, has now joined the list of secularising societies (Voas \& Chaves 2016). Of course, along with a drop in espoused affiliation, there has been a decline in the power held by religious institutions in these countries. While it would be unacceptable these days to interpret these changes in terms of inevitable and irreversible historical necessity, it is none-the-less necessary to provide an explanation. In particular, any evolutionarily-based account of religion must be able to explain what has happened in these societies to make religion no longer omnipresent and omnipotent. A further point that can be made is that since secularisation is a social phenomenon, any explanation of religiosity (and the society-wide patterns of changes it undergoes) must at least in part be a social explanation (Norris \& Inglehart 2004 for example). Evolutionary theorists, in particular, cannot limit themselves to consideration of human cognitive systems in order to explain secularisation. This much is obvious to anyone who remembers from Biology 101 that the phenotype is the product of the interaction between the genotype and the environment. 


\section{Just-so stories}

At the end of his introduction, Evans-Pritchard spells out his attitude to the various theories of religion that he goes on to examine in his book:

"I find all the theories we shall examine together no more than plausible and even, as they have been propounded, unacceptable in that they contain contradictions and other logical inadequacies, or in that they cannot, as stated, be proved either true or false, or finally, and most to the point, in that ethnographic evidence invalidates them." (Evans-Pritchard 1965, 18)

This is as true of the evolutionist theories, as of all the other accounts Evans-Pritchard looks at and therefore ought not be considered as a particular shortcoming of evolutionism as Evans-Pritchard saw it. Thus, for example, while he thinks quite highly of Durkheim's account of religion he, none-the-less, ends up concluding that it, also, is a just-so-story (Evans-Pritchard 1965, 64) - the term Kipling used for the fanciful stories he made up to 'explain' such things as how the tiger got its spots (it was grabbed by someone with dirty fingers). In other words, Evans-Pritchard felt that in the studies of religion he surveyed, be they evolutionist or not, theory ran largely free from any evidential basis. Given how broad the reach of this objection is according to Evans-Pritchard, it puts into question whether modern evolutionary approaches to explaining religion (or indeed any other approaches to the study of religion) avoid the problem, despite their fundamental differences to the evolutionist accounts Evans-Pritchard criticised. Therefore, to consider the relevance of Evans-Pritchard's objection to cognitive science of religion, it is necessary to examine the ways in which the balance between theory and evidence has changed over the last fifty years.

In so far as he looked at 'primitive' religion, the evidence that Evans-Pritchard primarily took into account was ethnographic - gathered by researchers who lived for months or even years with the peoples they studied, in order to gain a deep understanding of how their society functioned and what their beliefs meant. This evidence was then gathered and published as accounts of the beliefs and practices of the peoples, in order that it be available to other researchers. Naturally, Evans-Pritchard's own accounts of the life of the Azande and the Nuer are examples of just this kind of work. Over the last five decades, a great amount of high quality ethnographic work has been carried out, enormously increasing the amount of evidence that today's theories of religion can consider. At the same time, due to the nature of these studies, the evidence provided by them has much the same limitations as those pointed out by Evans-Pritchard. Thus, it would seem that no amount of ethnographic studies could by itself provide evidence as to the origin of religion that early theorists of religion were so concerned to discover - as Evans-Pritchard repeatedly pointed out. Apart from this kind of evidence, the study of 'primitive' religions could only rely upon archaeological material that was of limited relevance given that, infamously, beliefs and practices do not fossilise, making interpretation particularly difficult and contentious. 
The limitations on the available kinds of evidence were made all the more profound by Evans-Pritchard's philosophical view that the social was autonomous from psychological or biological considerations - an issue we will consider at length. Taken seriously, EvansPritchard's view would mean that biological or psychological evidence could not be used to reach conclusions about social phenomena. Modern scientific approaches to the study of religion, such as cognitive science of religion, do not generally agree that social phenomena have this level of autonomy, so that the range of potential evidence that is deemed to be relevant to theories of religion is much greater. In fact, the standard approach taken by cognitive science of religion is to theorize the existence of cognitive mechanisms whose functioning could explain some, often social, aspects of religious behaviour or belief. This multidisciplinarity goes hand-in-hand with the development of new research methods or their appropriation from other disciplines, and their application to the study of religion in general, as well as the empirical examination of the theorized mental mechanisms in particular.

The lines of evidence applied to the study of religion are now so numerous that it is necessary to choose just a few examples to illustrate the wealth of information available. Even if we only consider the evidence from traditional sources such as historical and ethnographic studies, the sheer volume of this material has grown exponentially over the last couple of decades, making it impossible for a modern researcher to be up-to-date with all of the individual studies that are of relevance. This has necessitated (as well as made possible) projects such as the Database of Religious History at UBC, headed by Edward Slingerland, which seek to collate the information from the various studies in a form that allows for easy analysis of patterns across societies and times (Slingerland and Sullivan 2017). The approach has already borne fruit. Researchers such as Russell Gray have used computational analysis to construct phylogenetic trees of religious traditions that have made it possible to justify fascinating conclusions regarding the development and significance of various religious practices (Gray, Drummond, and Greenhill 2009). Watts et al (2016) have been able to use phylogenetic analysis, for example, to show that human sacrifice has played a significant role in the development of complex, stratified societies. While this does not reveal the historical origin of religion per se, it does allow us to notice remarkable regularities in the historical development of religions that require further investigation and explanation.

Apart from the mostly qualitative data that has traditionally been gathered by ethnographers, the time since Evans-Pritchard's critique has witnessed an explosion in the amount of quantitative sociological data that is gathered world-wide and can be used by researchers to test hypotheses about religion. Foremost in terms of gathering high quality quantitative data from countries around the world are social science organisations such as the Pew Research Center and the World Values Survey. It is the data gathered by the latter of these two that has provided the basis for the existential security explanation for secularisation put forward by Norris and Inglehart (2004). Both the quantitative sociological studies and the historical databases represent the latest developments in the sort of 
rigorous 'comparative' work that Evans-Pritchard saw as woefully missing in the study of religion (Evans-Pritchard 1965, 10).

At the same time, data provided by historical and archaeological research is being reexamined in light of studies of the human cognitive system. In numerous cases, this provides novel, persuasive explanations for long-known but previously-unexplained phenomena. One example of this kind of work is provided by Ales Chalupa's (2014) work aimed at throwing new light on the Delphic Oracle by using modern psychology to formulate novel hypotheses concerning the mental and cultural mechanisms that may have underpinned the behaviour of the Pythiai during oracular sessions. It allows him to draw upon psychological work on modern examples of purported auguries to draw further comparisons as well as to exclude certain previously popular explanations on the grounds that they are psychologically implausible.

This leads us to consider a line of evidence that Evans-Pritchard would not have been happy to allow, but which has proved particularly significant in helping us develop a new understanding of religion. Experimental studies, carried out both in laboratory settings and in situ, have provided a wealth of novel, reliable information about religious phenomena that no amount of ethnography could have delivered. One recent example is the study by Xygalatas (2013) which has shown that pro-social behaviour can be increased by a natural religious setting, thereby helping us understand the connection between pro-sociality and religion - an issue of key significance for the study of religion that has proved particularly difficult to come to grips with. As always, any experimental research raises questions regarding its external validity. The sited study was carried out in situ, thereby avoiding many of the standard questions affecting laboratory studies, and measured pro-social behaviour using a means - the Dictator Game - which is much superior to questionnaire measures but which is still open to variant understanding in different cultural contexts (Henrich, Heine, and Norenzayan 2010), raising the potential objection that its significance might have been misunderstood in this context. Yet, external validity is not an issue that only affects experimental work - any work of ethnography faces the question of how far its insights apply beyond the immediate context that was investigated (LeCompte and Goetz 1982). This is true regardless of - or perhaps even because of - the level of thoroughness and detail of such a study. Any method that is used will raise particular issues. In general, the solution is to do more rather that less. The answer is not to avoid using some methods that can potentially cast light upon a phenomenon but, instead, to use a variety of them especially from across a variety of disciplines - in order to get a multi-dimensional picture of the phenomenon under study. By using a variety of methods, it becomes possible to identify and counteract the shortcomings of particular methodologies. This is the tactic used by cognitive science of religion, especially when it works in close cooperation with more traditional approaches.

The final line of evidence that Evans-Pritchard did not consider (indeed, could not consider) in his critique is the use of computers to simulate aspects of religions. An important recent example is the work of LeRon Shults and colleagues (2017) which has been able to 
identify conditions which lead to escalating religious violence. Agent-based modelling of social phenomena is of particular significance to Evans-Pritchard's concerns regarding what evidence is available to support theories about religion. The reason is that researchers such as Shults are in effect modelling how changes at the individual, psychological level come to lead to differences at the social level by creating computer models of individuals (based on empirical evidence or testing particular theoretical claims), letting them interact with each other and observing what social level patterns appear out of these interactions. This makes it possible to track the connections between the social and psychological, in effect showing the ways in which the social level is definitely not autonomous of psychological considerations.

Given this wealth of novel and greatly expanded sources of evidence, it might seem that the worries Evans-Pritchard had about theory outpacing evidence should no longer hold. The point can be best seen by considering questions regarding the historical genesis of religion (or its features) that Evans-Pritchard deemed beyond the ken of science. We now have to hand a wealth of data regarding the history of religion, data that has been organised in databases, providing a rich basis for phylogenetic analyses that help to make plausible probabilistic claims about the appearance and spread of particular features of religion. Something like this approach has been taken by recent efforts to determine whether moralising religions predated complex societies - an issue of central import to the Big Gods hypothesis (Norenzayan 2013) and which is currently hotly debated (Whitehouse et al. 2019; Beheim et al. 2019; Savage et al., n.d.). The hypotheses put forward in this manner can be further tested by using quantitative sociological data and the results of experimental studies to build realistic computer models of individuals and determining to what degree the relevant circumstances lead to similar outcomes when modelled on a computer. Taken together, such a multipronged effort promises to bring together questions of psychological and historical genesis of religion and thereby provide us with a scientific basis for determining answers to questions Evans-Pritchard would not have thought answerable - much in the manner that spectrography allowed astronomers to determine the chemical constitution of distant stars only a few decades after August Comte claimed science would never determine it.

Undeniably, the data available to theoreticians of religion and the methods that can be used to gather that data are now far beyond those Evans-Pritchard saw theory of religion as reliant upon. Considered by itself, however, this does not mean that the critiques he raised against the theories of his day do not similarly disturb those of today. To see whether that is the case, it is necessary to also consider today's theories, themselves. In particular, it is necessary to consider what evidence is required to justify evolutionary theories regarding religion. This is because, as we will see, they have a particularly heavy evidential burden due to their deep foundations.

Evans-Pritchard on a number of occasions claimed that various theories of religion were just-so stories - not much more than the fanciful narratives that Kipling playfully invented to post-hoc 'explain' the traits of animals. The same term has been used more recently by 
Stephen Jay Gould (1978), among many others, to criticise adaptationist evolutionary explanations of human behaviour, particularly as proposed by evolutionary psychology. In both cases, the imputation is that the theories put forward, while prima facie plausible, are neither necessitated by the data nor used to develop novel, testable predictions. The issue, identified by Al-Shawaf et al (2019), appears to be that historical claims can easily appear to be unverifiable. This seems to be precisely the issue Evans-Pritchard has with claims about the historical genesis of religion and it is very much an issue for evolutionary explanations because of the way they rely upon claims regarding evolutionary history. As we have seen, given the appropriate methodology, however, historical claims have implications that can be currently tested. This is exactly the conclusion drawn about testing of the Big Gods hypothesis discussed above and it is the same point that Al-Shawaf and colleagues make.

To see in detail how evolutionary theories of religion necessarily come to make historical claims, it is useful to consider Niko Tinbergen's (1963) four questions (see Bateson and Laland 2013b; Nesse 2013; Bateson and Laland 2013a for a recent discussion), which have to be answered in order to provide a full explanation of biological traits (including behavioural traits) in general - with human religion being understood as an example of that. Two of Tinbergen's questions do not raise the need to consider the evolutionary history of religion, dealing with proximate causes. The first of these is the question of what is the mechanism (or mechanisms) causing the trait. This is the question that is most focused upon by much of the work in cognitive science of religion - the identification of specific cognitive (and cultural) mechanisms that generate religious beliefs and practices. It has been proposed, for example, that the human propensity to postulate supernatural entities such as ghosts and fairies is caused by a mental module called the hyperactive agency detection device (HADD) that leads people to overinterpret events due to agential activity (Guthrie 1993) - with the cultural context typically determining many aspects of the interpretation. The second of these proximate questions, that do not necessarily involve considerations of evolution, is that of ontogeny - how the trait develops in individuals. This question is all too often not dealt with in enough detail. It is essential, however, in that it links the mechanisms with the evolutionary questions by showing how it is that the trait comes to be reliably generated by the interaction of the current environment and the genotype which is the end product of evolutionary change. In the case of HADD-caused supernatural beliefs, an answer to this question would involve at a minimum demonstrating how differences in genetics and in the environment during development affect the extent of such beliefs. One variable that is thought to play a vital role here is the extent to which an individual feels anxiety during development.

Neither of these questions require historical evidence - the mechanisms as well as ontogeny can be studied experimentally on the individual subjects. The issue is more complex with the evolutionary (or ultimate) questions that Tinbergen identified. The first of the evolutionary questions concerns the current utility or adaptive significance of the trait - whether it is functional, or has its current form for some other evolutionary reason, such as due to genetic drift. Current utility might appear to not be a matter of history 
and determinable without the need to look at historical evidence. At best, however, this is the case for functional traits and even then only given particular definitions of biological function. Traits shaped by genetic drift are the result, for example, of random fluctuations in the occurrence of particular alleles that are not under selective pressure - this process becoming particularly significant in small populations. In addition, the biological function is often defined as the reason why a particular trait has become common in a population. In both cases, determining the adaptive significance of the trait involves historical questions. At the same time, answering those questions does not necessarily require examining what might be thought of as 'historical' evidence. This is because it is possible to analyse genomes of current populations to identify genes that have been under selection pressure (Kosiol and Anisimova 2019) - the statistical methods required being very closely connected to the phylogenetic analyses carried out by Russell Gray and colleagues and discussed earlier.

In the case of religious beliefs and practices, it is most common for them to be considered to be cognitive by-products - the nonfunctional side-effects of traits that are functional. This is exactly the case with the tendency to postulate supernatural entities, that is the side-effect of the HADD. The capacity to detect agents seems clearly functional, given their significance to survival and procreation. Similarly, it makes sense that it would be functional for the device that detects the agents to be hyperactive - given that false positives will generally be far less costly than false negatives (Haselton and Buss 2000). The numerous false positives, however, will necessarily generate a great deal of pseudo-evidence for the activity of supernatural entities - thereby ensuring that such beliefs are common as a by-product. As we will see, however, at this stage, much of this account is conjecture with concrete evidence lacking.

This leaves us with the last of Tinbergen's questions, that of the phylogenetic history of the trait - in other words the evolutionary history of how the trait changed over the preceding generations. Clearly, this is very much a historical question - an answer to it would require spelling out the forms the precursors of the trait took potentially over millions of years. Finding evidence for any answers would appear to be particularly difficult in the case of a behavioural trait. Yet, comparative data from other species makes it possible to determine some of that history, the relevant statistical analyses again being connected to examples of phylogenetic analysis considered earlier. In the case of the HADD, two hypotheses may be put forward. The first is that this device is connected to the hypersocial lifestyle of humans and that, therefore, we would not find it even in our closest ancestors such as chimpanzees. The second (much more plausible) alternative is that the need to identify other agents is common to all animals and that, therefore, the trait is likely to have a very long evolutionary history and be present in many of the living species of animals. This does not mean, of course, that it generates the same kinds of supernatural beliefs in other animals as it is thought to generate in humans.

Importantly, despite it now being more than several decades since the hyperactive agency detection device was proposed as a hypothesis, and despite the efforts of numerous researchers, there is still a relative lack of solid evidence either for its existence or for 
its role in the generation of supernatural beliefs. This is due to a fundamental mismatch between how easy it is propose plausible evolutionary explanations and how difficult it is to provide evidence for them. Evolutionary theory provides a rich plethora of possible kinds of explanations that earlier approaches could not hope for. Unfortunately, identifying and justifying the correct one requires not just the efforts of a single research team but, given the multifarious methods required, the efforts of a number of research teams from a range of scientific disciplines all cooperating towards producing that evidence. What makes it worse is that, given how radically counterintuitive evolutionary biology can be, an initially plausible hypothesis may easily be fundamentally mistaken. There is indeed therefore something to be said for calling evolutionary theories of religion just-so stories. It would be wrong to conclude, however, that Evans-Pritchard's negative evaluation of theories of religion, quoted at the start of this section, applies to modern evolutionary theories of religion. They are in general neither falsified nor unfalsifiable.

As we have seen, Evans-Pritchard was particularly concerned about the capacity to provide evidence for historical claims, and evolutionary theories of religion necessarily need to make such claims. Such claims are not made, however, in an evidential vacuum - they have consequences that are testable using widely available methods, be they claims about the historical development of religion or the evolutionary history of the cognitive traits that underpin our religious beliefs and behaviours. The same holds for the other claims that are made by theories put forward within cognitive science of religion. The hyperactive agency-detection device was chosen as an example for a reason - it is relatively amenable to the kind of critique that Evans-Pritchard put forward because of the weakness of the existing evidence for it. It is not unfalsifiable, however, it has observational consequences that have begun to be investigated, including the link between supernatural beliefs and a tendency to illusory agency detection (Elk 2013). Other theories, such as the role of religion in prosociality, are far better supported (Xygalatas and Lang 2017).

Two more points should be raised. If it is the case that understanding religion requires explanations that involve evolved traits, then it is only by looking at all of the questions that Tinbergen asked that we can hope to achieve something like a fully-rounded understanding of religion. This means, however, that anything but an evolutionary approach of religion is hamstrung, both in terms of the explanatory theories it can put forward and in terms of the methods that it can use to find evidence for or against them. Finally, as the discussion of the hyperactive agency-detection device should have made clear, the theories of religion that are put forward by cognitive science of religion are fundamentally different, not just in terms of relying upon evolutionary biology, but also in terms of what they are theories of - the kinds of entities that they concern. In particular, in an important sense they are not theories of religion. This is the issue that will be discussed at length in the following section. 


\section{The social and the psychological}

Evans-Pritchard divides the theories of religion that he criticises into the social and the psychological. The psychological theories, which Evans-Pritchard criticises first, focus on the psychological processes that are involved in religion, an approach which he thinks is fundamentally mistaken:

If religion is characterized by the emotion of fear, then a man fleeing in terror from a charging buffalo might be said to be performing a religious act; and if magic is characterized by its cathartic function, then a medical practitioner who relieves a patient's anxiety, on entirely clinical grounds, might be said to be performing a magical one. (Evans-Pritchard 1965, 44)

Given that most accounts in cognitive science of religion are concerned with the cognitive mechanisms that play a role in religion, the issue is prima facie relevant to CSR. If belief in supernatural entities is caused, for example, by illusory agency detection resulting from the hyperactive activity of the agency-detection device, is it the case that any illusory entity that the HADD leads us to believe in is supernatural or even religious?

Such a claim would be very hard to support given the quotidian experience of thinking that we have heard someone in the next room when we are alone in the house, and cognitive approaches to the study of religion do not require it. Ann Taves (2009) makes the essential point most clearly, arguing that instead of trying to "characterise religion" we should disaggregate the concept of religion and instead seek to understand the interaction of the psychological and social processes that determine whether something is deemed to be religious. Thus, understanding how the HADD produces belief in supernatural entities is not intended to provide a characterisation of religion but, simply, to explain one of the mechanisms that play a role in the appearance and transmission of supernatural beliefs. The HADD might produce belief in illusory entities but it is only because of interactions with other social and psychological mechanisms that these entities come to be characterised as religious or magical.

Evans-Pritchard $(1965,18)$ explicitly allowed the kind of explanatory pluralism that is a core element of CSR. He did not, however, seem to fully appreciate that it provides a response to some of objections he raised. An attempt to explain religion in purely psychological terms would necessarily be a failure given that cultural differences between particular religious traditions, and social changes such as secularisation, cannot be fully explained without reference to social factors or, in many cases, cultural history. At the same time, a purely sociological account of the sort Evans-Pritchard seemed to favour at times, would not be able to explain why particular kinds of beliefs and practices were repeatedly taken up and reproduced across cultures and times. Religion might be independent of particular individual minds, as Evans-Pritchard $(1965,54)$ claimed, but this does not mean that it is not dependent for its existence on those minds considered together or that it is not shaped by their common traits. 
There are two main kinds of explanatory accounts of religion pursued within CSR. The first characterises supernatural beliefs and practices as cognitive by-products, the coincidental results of the functioning of cognitive systems that exist to serve some other function. The hyperactive agency-detection device is one example of such an account. At the same time, many researchers are considering the role that some elements of religion appear to play in motivating pro-social behaviour. These two kinds of accounts at first might appear to simply recapitulate the psychological/sociological distinction in Evans-Pritchard. Both kinds of accounts do not eschew, however, either psychological or sociological elements and, in addition, the two kinds of accounts have been combined by a number of researchers who argue that religious traditions have recruited cognitive by-products to help motivate prosocial behaviour (Atran and Henrich 2010; Talmont-Kaminski 2014; Norenzayan 2013).

What Evans-Pritchard is more likely to have thought profoundly objectionable in the approach Taves proposes, however, is her suggestion that the concept of religion should be disaggregated, or to use another word - reduced. Taves is adamant that the correct way to study religion is in terms of processes and mechanisms that are not 'religious' in and of themselves - a candid rejection of the view of religion as sui generis that Evans-Pritchard and many other mid-twentieth-century researchers held.

Evans-Pritchard $(1965,17)$ held that a reductionist approach aimed at providing a causal explanation of religion is motivated by the desire to show that religious belief is false. He thought that the phenomenological method is the properly scientific one that avoids theological questions. However, while the attempt to explain religion in naturalist terms may be necessary to justify an atheist philosophy, it is hardly sufficient. Demonstrating that human religious beliefs have their basis in human cognition does not show them to be false any more than showing that someone guessed the lottery numbers demonstrates that they will lose. All it does is undermine the argument that the universality of religious beliefs renders them probable. In effect, a survey of CSR researchers would find that they follow Evans-Pritchard's dictum not to be concerned, qua CSR researchers, with the truth or falsity of religious thought - even though their interest in the study of religion is often motivated by a range of both negative and positive theological beliefs (see McCauley and Lawson 2017 for a detailed discussion).

The naturalist approach favoured by Taves and cognitive scientists of religion in general can be understood as a reaction to what was seen as a failure of nerve in the study of religion (Wiebe 1984; Arnal, Braun, and McCutcheon 2014; Ambasciano 2019). Taves, herself, explains it in Durkheimian terms as an attempt to maintain the sanctity of religion (Taves 2009, 33-34). The line of thought can be pursued even further, however. As Talmont-Kaminski (2013) argues, deeming religious claims too sacred to examine closely or to investigate as natural phenomena is necessary to maintain their pro-social function. This is because this function is not connected to their truth. Accepting religious claims on the basis of their accuracy would in all likelihood eliminate those that best motivate prosocial behaviour - unlike other kinds of beliefs in whose case their function is connected to their accuracy. The reason why it has been so difficult to study religion scientifically 
can be traced to the conflicting ways in which scientific and religious institutions make use of the human capacity to determine whether to accept the claims made by others, i.e. our capacity to maintain epistemic vigilance (Sperber et al. 2010). Normally, when evaluating claims we consider both the plausibility of the content of the claims (content vigilance) as well as the trustworthiness of their source (source vigilance). Religious and scientific institutions tend, however, to constrain and direct those tendencies to suit their purpose. Religious institutions promote source vigilance in order to create traditions of belief that can become independent of their accuracy - as required by their function also being independent of their accuracy. Scientific institutions favour content vigilance, allowing claims to be evaluated much more on their own merits, and thereby abjuring the very same kinds of traditions of beliefs that religions require. This difference in the kinds of vigilance promoted leads to a direct clash of epistemic standards when attempts are made to scientifically study religious claims (Talmont-Kaminski 2020). Understood in those terms, the reductionist approach, far from being objectionable to Evans-Pritchard, is perhaps surprisingly the approach to be taken in order to avoid allowing one's religious views to determine one's scientific positions.

There is an altogether different and much more constructive way to consider reductionism in the scientific study of religion. According to Evans-Pritchard, religion is a theoretically deep concept that a scientific account must explain in order to be adequate. It is in this sense that the accounts Evans-Pritchard considers are theories of religion. Indeed, if religion is sui generis then, in some way, religion must play an integral role in whatever explanations are offered. Taves does not consider religion to be either explanans or explanandum. What is to be explained are particular beliefs, practices or experiences that have been traditionally deemed to be religious. Their explanations are causal and built out of particular cognitive, cultural and evolutionary processes and mechanisms. Religion does not really enter into the picture, turning out to be for the most part theoretically inconsequential. In fact, a number of cognitive scientists of religion, such as Pascal Boyer (2010), argue that religion is a concept that does not refer to any particular entity in the real-world, religion being more of a grab bag of independently existing phenomena.

The resulting picture is anything but simplistic. A multidisciplinary approach to understanding 'religious' phenomena, quite naturally calls for an explanatory picture that must describe the phenomena on a number of levels - a 'causal thicket' of the type described by Wimsatt (2007). Certainly, both sociological and psychological mechanisms have to be part of such an account. Indeed, given the necessary complexity of even the most basic sketch of an explanation that a modern evolutionary approach to religion requires, one could justifiably claim that it is the sui generis accounts that are in truth 'reductionist' in that they effectively reject how religion is interconnected with a great range of social and psychological aspects of human life at the most fundamental, mechanistic level. 


\section{Conclusions}

The method pursued in this article involving looking at Evans-Pritchard's objections that could be seen as relevant to modern evolutionary approaches to religion has been to start with the specific and pan out to the most basic.

The particular objections Evans-Pritchard raised against evolutionist theories of religion did not directly impact approaches pursued within cognitive science of religion because these approaches reject the theoretical claims that were so problematic in the case of evolutionism. The issues Evans-Pritchard raised maintain their relevance, however, even though their significance is much altered by the very different context of modern scientific study of religion. This pattern of continued but transformed significance recurred both in the case of questions of the relationship between theory and evidence and in the case of the stance taken with regard to naturalism. Modern evolutionary approaches to religion do not ignore Evans-Pritchard. It would be fairer to say that they have taken on-board his criticisms but, in so doing, the points he raised have co-evolved with the modern evolutionary theories of religion in ways that he could not have predicted. One point of clear consensus remains:

"If I can persuade you that much is still very uncertain and obscure, my labour will not have been in vain. You will then not be under any illusion that we have final answers to the questions posed" (Evans-Pritchard 1965, 4)

\section{Bibliography}

Al-shawaf, Laith, Kareem Zreik, and David M Buss. 2019. “Thirteen Misunderstandings about Natural Selection." In Encyclopedia of Evolutionary Psychological Science, edited by T. K. Shackleford and V. A. WeekesShackleford. Springer. https://doi.org/10.1007/978-3-319-16999-6.

Ambasciano, Leonardo. 2019. An Unnatural History of Religions. London \& New York: Bloomsbury Academic. Arnal, WE, W Braun, and RT McCutcheon, eds. 2014. Failure and Nerve in the Academic Study of Religion. Sheffield: Equinox Publishing.

Atran, Scott, and J. Henrich. 2010. "The Evolution of Religion: How Cognitive by-Products, Adaptive Learning Heuristics, Ritual Displays, and Group Competition Generate Deep Commitments to Prosocial Religions." Biological Theory 5 (1): 18-30.

Bateson, Patrick, and Kevin N. Laland. 2013a. "On Current Utility and Adaptive Significance: A Response to Nesse." Trends in Ecology and Evolution. https://doi.org/10.1016/j.tree.2013.10.009.

Bateson, Patrick, and Kevin N. Laland. 2013b. “Tinbergen's Four Questions: An Appreciation and an Update.” Trends in Ecology and Evolution. https://doi.org/10.1016/j.tree.2013.09.013.

Beheim, Bret, Quentin Atkinson, Joseph Bulbulia, William Gervais, Russell Gray, Joseph

Henrich, Martin Lang, et al. 2019. "Corrected Analyses Show That Moralizing Gods Precede Complex Societies but Serious Data Concerns Remain In Reply to 'Complex Societies Precede Moralizing Gods throughout World History.” https://doi.org/10.31234/osf.io/jwa2n. 
Boyer, Pascal. 2010. The Fracture of An Illusion: Science And The Dissolution Of Religion. Vandenhoeck \& Ruprecht. http://www.amazon.com/Fracture-Illusion-Dissolution-Religion-Naturwissenschaft/ $\mathrm{dp} / 3525569408$.

Chalupa, Aleš. 2014. "Pythiai and Inspired Divination in the Delphic Oracle: Can Cognitive Sciences Provide Us with an Access to 'Dead Minds'?" Journal of Cognitive Historiography 1: 24-51. https://doi. org/10.1558/jch.v1i1.24.

Elk, Michiel van. 2013. "Paranormal Believers Are More Prone to Illusory Agency Detection than Skeptics." Consciousness and Cognition 22 (3): 1041-46. https://doi.org/10.1016/j.concog.2013.07.004.

Evans-Pritchard, Edward Evan. 1965. Theories of Primitive Religion. Clarendon Press. http://books.google. com/books?id=ewgdv5eNLT0C\&pgis=1.

Gould, Stephen Jay. 1978. "Sociobiology: The Art of Storytelling.” New Scientist 80 (1129): 530-33.

Gray, R. D., A. J. Drummond, and S. J. Greenhill. 2009. "Language Phylogenies Reveal Expansion Pulses and Pauses in Pacific Settlement.” Science 323 (5913): 479-83. https://doi.org/10.1126/science.1166858.

Guthrie, Stewart. 1993. Faces in the Clouds. New York: Oxford University Press, USA.

Haselton, Martie G, and David M Buss. 2000. "Error Management Theory: A New Perspective on Biases in Cross-Sex Mind Reading." Journal of Personality and Social Psychology 78 (1): 81-91.

Henrich, Joseph, Steven J Heine, and Ara Norenzayan. 2010. “The Weirdest People in the World?” Behavioral and Brain Sciences 33 (2-3): 61-83. https://doi.org/10.1017/S0140525X0999152X.

Kosiol, Carolin, and Maria Anisimova. 2019. "Selection Acting on Genomes." In Methods in Molecular Biology, 1910:373-97. Humana Press Inc. https://doi.org/10.1007/978-1-4939-9074-0_12.

LeCompte, Margaret, and Judith Preissle Goetz. 1982. "Problems of Reliability and Validity in Ethnographic Research.” Review of Educational Research 52 (1): 31. https://doi.org/10.2307/1170272.

McCauley, Robert N., and E. Thomas Lawson. 2017. Philosophical Foundations of the Cognitive Science of Religion: A Head Start. Bloomsbury Academic. https://doi.org/10.5040/9781350030343.

Nesse, Randolph M. 2013. “Tinbergen's Four Questions, Organized: A Response to Bateson and Laland.” Trends in Ecology and Evolution. https://doi.org/10.1016/j.tree.2013.10.008.

Norenzayan, Ara. 2013. Big Gods: How Religion Transformed Cooperation and Conflict. Princeton: Princeton University Press.

Norris, Pippa, and Ronald Inglehart. 2004. Sacred and Secular: Religion and Politics Worldwide. Cambridge, UK: Cambridge University Press.

Savage, Patrick E., Harvey Whitehouse, Pieter François, Thomas E. Currie, Kevin C. Feeney, Enrico Cioni, Rosalind Purcell, et al. n.d. "Reply to Beheim et Al.: Reanalyses Confirm Robustness of Original Analyses." SocArXiv Preprint. https://doi.org/10.31235/osf.io/xjryt.

Shults, F LeRon, Ross Gore, Wesley J Wildman, Justin E Lane, Chris Lynch, and Monica Toft. 2017. "Mutually Escalating Religious Violence: A Generative and Predictive Computational Model." SSC Conference Proceedings, no. September: 1-12.

Slingerland, Edward, and Brenton Sullivan. 2017. "Durkheim with Data: The Database of Religious History." Journal of the American Academy of Religion 85 (2): 312-47. https://doi.org/10.1093/jaarel/lfw012.

Sperber, Dan, Fabrice Clement, Christophe Heintz, Olivier Mascaro, Hugo Mercier, G. Origgi, and Deirdre Wilson. 2010. "Epistemic Vigilance." Mind \& Language 25 (4): 359-393. http://onlinelibrary.wiley.com/ doi/10.1111/j.1468-0017.2010.01394.x/full. 
Talmont-Kaminski, Konrad. 2013. "For God and Country, Not Necessarily for Truth: The Nonalethic Function of Superempirical Beliefs." The Monist 96 (3): 447-61.

Talmont-Kaminski, Konrad. 2014. Religion as Magical Ideology: How the Supernatural Reflects Rationality. Abingdon, Oxon: Routledge.

Talmont-Kaminski, Konrad. 2020. "Epistemic Vigilance and the Science/Religion Distinction.” Journal of Cognition and Culture forthcoming.

Taves, Ann. 2009. Religious Experience Reconsidered: A Building Block Approach to the Study of Religion and Other Special Things. Princeton University Press.

Tinbergen, Nikolaas. 1963. "On Aims and Methods in Ethology." Zeitschrift Für Tierpsychologie 20: 410-33.

Voas, David. 2008. “The Rise and Fall of Fuzzy Fidelity in Europe." European Sociological Review 25 (2): 155-68. https://doi.org/10.1093/esr/jcn044.

Voas, David, and Mark Chaves. 2016. "Is the United States a Counterexample to the Secularization Thesis?" American Journal of Sociology 121 (5): 1517-56. https://doi.org/10.1086/684202.

Watts, Joseph, Oliver Sheehan, Quentin D. Atkinson, Joseph Bulbulia, and Russell D. Gray. 2016. "Ritual Human Sacrifice Promoted and Sustained the Evolution of Stratified Societies.” Nature 532 (7598): 228-31. https://doi.org/10.1038/nature17159.

Whitehouse, Harvey, Pieter François, Patrick E. Savage, Thomas E. Currie, Kevin C. Feeney, Enrico Cioni, Rosalind Purcell, et al. 2019. "Complex Societies Precede Moralizing Gods throughout World History." Nature. Nature Publishing Group. https://doi.org/10.1038/s41586-019-1043-4.

Wiebe, Donald. 1984. "The Failure of Nerve in the Academic Study of Religion.” Studies in Religion/Sciences Religieuses 13 (4): 401-22. https://doi.org/10.1177/000842988401300403.

Wimsatt, William C. 2007. Re-Engineering Philosophy for Limited Beings: Piecewise Approximations To ... Cambridge, MA: Harvard University Press.

Xygalatas, Dimitris. 2013. "Effects of Religious Setting on Cooperative Behavior: A Case Study from Mauritius." Religion, Brain and Behavior 3 (2): 91-102. https://doi.org/10.1080/2153599X.2012.724547.

Xygalatas, Dimitris, and Martin Lang. 2017. "Prosociality and Religion.” Mental Religion: The Brain, Cognition, and Culture, 119-33. 